\title{
Explicit Adaptive Time-Delay Compensation for Bilateral Teleoperation
}

\author{
Khalid Abidi ${ }^{1}$, Yildiray Yildiz ${ }^{2}$, Bekir Emre Korpe ${ }^{2}$ \\ 1. Newcastle University International Singapore, Nanyang Polytechnic, 569830 Singapore \\ E-mail: Khalid.Abidi@newcastle.ac.uk \\ 2. Bilkent University, Department of Mechanical Engineering, Cankaya, Ankara 06800, Turkey \\ E-mail: yyildiz@bilkent.edu.tr, bekir.korpe@ug.bilkent.edu.tr
}

\begin{abstract}
This paper proposes a control framework that addresses the destabilizing effect of communication timedelays and system uncertainties in telerobotics, in the presence of force-feedback. Force feedback is necessary to obtain transparency, which is providing the human operator as close a feel as possible of the environment where the slave robot is operating. Achieving stability and providing transparency are conflicting goals. This is the major reason why currently a very few, if at all, fully operational force feedback teleoperation devices exist except for research environments. The proposed framework handles system uncertainty with adaptation and communication time delays with explicit delay compensation. The technology that allows this explicit adaptive time-delay compensation is inspired by MIT's Adaptive Posicast Controller.
\end{abstract}

Key Words: Teleoperation, adaptive control, time delay systems

\section{INTRODUCTION}

One of the most creative solutions to the stability problem in force feedback teleoperation is using scattering and passivity theories [1-3]. These methods provide stability independent of the communication time delay value. The main problem with these approaches, however, is that transparency may be sacrificed to obtain stability. There have been studies to obtain better transparency using these approaches [4-10]. One of the approaches to provide increased transparency is using predictive control [2,11]. In this approach the main idea is predicting slave robot's future behavior using known system dynamics and feeding it back to the master robot. Smith predictors are one common approach used for this purpose [12]. The problem with this approach is that Smith predictors are known to be sensitive to modeling errors in the known system dynamics and errors in the known amount of the time delay. Therefore, modeling uncertainties or uncertainties caused by actuator degradation, parameter changes due to temperature variation and component aging can cause dangerous instabilities if this method is not used with caution. A different approach proposed in the literature is to use local impedence controllers to stabilize the slave and master robots improving robustness to time delay, [13]. This approach is also sensitive to modeling errors and does not preserve transparency. Another approach proposed in the literature to increase transparency and stability in the presence of time-delays and uncertainties is employing adaptive control [14]. In this approach, each manipulator has its local adaptive controller to address modeling uncertainties. The controller in [14] is designed in continuous time and a switching coefficient is used which is set according to free-motion or contact scenarios. This switching may cause erratic behavior if not handled properly and it requires ef- fort to obtain smooth switching between operation conditions. The proposed approach in this paper does not require switching. In addition, the proposed controller is designed in discrete time which eliminates inaccuracies emanating from discrete approximations of continuous time controllers in real applications.

In this work, we propose a telerobotics framework that may lead the way towards making fully operational, stable bilateral teleoperation a possibility without sacrificing transparency. We build upon the earlier successful research results, presented above, by eliminating the need for precise system models and eliminating the sacrifice of transparency by developing an adaptive controller in tandem with an explicit delay-compensating controller. Adaptive Posicast Controller (APC) $[15,16]$ is at the heart of this work. The main contribution of this paper is merging explicit delay compensation and adaptation in the teleoperation framework, in a mathematically rigorous way. There are key distinctions of this work compared to earlier studies. Firstly, unlike most passivity based approaches, transparency is not sacrificed for stability and there is no need for precise plant models. Secondly, unlike earlier adaptive approaches, there is no need for persistently exciting (rich) input excitations for parameter identification; there is no need for switching between controllers and the design is conducted in discrete time. Thirdly, the time-delay in the system will be explicitly compensated instead of building a control system that is robust to delays. These distinctions provide stability and increased transparency at the same time. The main approach, explicit adaptive delay compensation, will be achieved by employing a discrete adaptive controller locally and explicit time delay compensating controller inspired by the discrete-time version of APC [17]. APC is experimentally proven to be very ef- 
fective by the author Yildiz and his collaborators for automotive control problems [18-22]. To see a list of delaycompensating controllers and an investigation of predictive laws for delay perturbations, see [23].

The organization of the paper is as follows: The problem formulation and fixed controller design are presented in Section 2 and Section 3, respectively. Adaptive controller design is introduced in Section 4 followed by a summary in Section 5.

\section{PROBLEM FORMULATION}

Consider the Euler-Lagrange equations [25], [10] of an $n_{m}$-link master and $n_{s}$-link slave teleoperation system with a description given as

$$
\begin{aligned}
& M_{m}\left(q_{m}\right) \ddot{q}_{m}+C_{m}\left(q_{m}, \dot{q}_{m}\right) \dot{q}_{m}+g_{m}\left(q_{m}\right)=\tau_{m}(t) \\
& +J_{m}^{T}\left(q_{m}\right) f_{h}(t)
\end{aligned}
$$$$
M_{s}\left(q_{s}\right) \ddot{q}_{s}+C_{s}\left(q_{s}, \dot{q}_{s}\right) \dot{q}_{s}+g_{s}\left(q_{s}\right)=\tau_{s}(t)-J_{s}^{T}\left(q_{s}\right) f_{e}(t)
$$

where $q_{m} \in \Re^{n_{m} \times 1}, q_{s} \in \Re^{n_{s} \times 1}$ are the joint displacement vectors, $\dot{q}_{m} \in \Re^{n_{m} \times 1}, \dot{q}_{s} \in \Re^{n_{s} \times 1}$ are the joint velocity vectors, $\tau_{m}(t) \in \Re^{n_{m} \times 1}, \tau_{s}(t) \in \Re^{n_{s} \times 1}$ are the joint torque vectors, $M_{m}\left(q_{m}\right) \in \Re^{n_{m} \times n_{m}}, M_{s}\left(q_{s}\right) \in \Re^{n_{s} \times n_{s}}$ are the inertia matrices, $C_{m}\left(q_{m}, \dot{q}_{m}\right), C_{s}\left(q_{s}, \dot{q}_{s}\right)$ are the Centripetal and Coriolis torques matrices, $g_{m}\left(q_{m}\right) \in \Re^{n_{m} \times 1}, g_{s}\left(q_{s}\right) \in \Re^{n_{s} \times 1}$ are the gravitational torque vectors, $J_{m}\left(q_{m}\right) \in \Re^{l \times n_{m}}, J_{s}\left(q_{s}\right) \in \Re^{l \times n_{s}}$ are the Jacobian matrices, $f_{h} \in \Re^{l \times 1}$ is the operator hand force vector and $f_{e} \in \Re^{l \times 1}$ is the contact force vector on the slave robot. In this work, the master and slave parameters are assumed to be uncertain. The Euler-Lagrange equations (1) and (2) have the following useful property due to their structure [6].

Property 1. The Lagrangian dynamics are linearly parametrizable [26] which gives the form

$$
M(q) \ddot{q}+C(q, \dot{q}) \dot{q}+g(q)=Y(q, \dot{q}, \ddot{q}) \theta=\tau(t)
$$

where $\theta$ is a constant p-dimensional vector of parameters and $Y(q, \dot{q}, \ddot{q}) \in \Re^{n \times p}$ is the matrix of known functions of the joint displacements and their derivatives.

In this implementation the operator hand force is modeled as

$$
f_{h}(t)=\alpha_{0}-K_{h} x_{m}(t)-B_{h} \dot{x}_{m}(t)
$$

where $\alpha_{0}$ represents a constant non-passive force exerted by the operator resisted by the passive component $-K_{h} x_{m}(t)-B_{h} \dot{x}_{m}(t)$, and $x_{m}(t), \dot{x}_{m}(t)$ are the diplacement and velocity vectors of the master robot end-effector [6]. The contact force on the slave robot is modeled as a passive force of the form

$$
f_{e}(t)=K_{e} x_{s}(t)+B_{e} \dot{x}_{s}(t)
$$

where $x_{s}(t), \dot{x}_{s}(t)$ are the displacement and velocity vectors of the slave robot end-effector. Note that the matrices $K_{h}, K_{e}$ and $B_{h}, B_{e}$ represent uncertain stiffness and damping of the operator and the environment. It is possible to rewrite the force models (3) and (4) in the parameterized form as follows

$$
f_{h}(t)=\alpha_{0}-K_{h} x_{m}(t)-B_{h} \dot{x}_{m}(t)=\alpha_{0}-\Theta_{h} \chi_{m}
$$

and

$$
f_{e}(t)=K_{e} x_{s}(t)+B_{e} \dot{x}_{s}(t)=\Theta_{e} \chi_{s}
$$

where $\Theta_{h} \in \Re^{l \times 2 l}, \Theta_{e} \in \Re^{l \times 2 l}$ are constant matrices of the uncertain parameters and $\chi_{m, k}=\left[x_{m, k}^{T}, \dot{x}_{m, k}^{T}\right]^{T} \in$ $\Re^{2 l}, \chi_{s, k}=\left[x_{s, k}^{T}, \dot{x}_{s, k}^{T}\right]^{T} \in \Re^{2 l}$.

Combining (1), (2) and Property 1 the system reduces to the form

$$
\begin{gathered}
Y_{m}\left(q_{m}, \dot{q}_{m}, \ddot{q}_{m}\right) \theta_{m}=\tau_{m}(t)+J_{m}^{T}\left(q_{m}\right) f_{h}(t) \\
Y_{s}\left(q_{s}, \dot{q}_{s}, \ddot{q}_{s}\right) \theta_{s}=\tau_{s}(t)-J_{m}^{T}\left(q_{s}\right) f_{e}(t)
\end{gathered}
$$

Technically, transparency is defined as:

$$
\begin{gathered}
f_{h}(t)=f_{e}(t) \\
x_{m}(t)=x_{s}(t)
\end{gathered}
$$

According to this definition, in a transparent system, the slave tracks the master and at the same time the operator feels the external force acting on the slave robot. This is desired in many applications but there may be situations where a slightly different structure is preferred. For example, during a free motion, i.e. when the slave robot is moving freely without any contact to its environment, the operator should not feel anything according to the above transparency definition. However, there may be situations where this may result in dangerous behavior: If the operator feels no resistance, he/she can move the master robot in a way that can saturate the slave robot actuators and cause the slave robot move in an unpredictable way. In addition, feeling nothing may not be desired by the operator. He/she may require a feel of inertia in his/her hands to "understand better" the tool (slave robot) he/she is using to use it in a more precise and controlled manner. Similarly, during contact with the environment, the operator may want the feel of the tool he/she using together with the effect of the external environmental force acting on it. For example, a surgeon may desire to feel the inertia of the cutting apparatus he/she is using together with the effect of the tissue on the apparatus. We develop the proposed telerobotics framework based on these considerations, so that the slave follows the master and the operator feels the virtual control force that is applied to the modified slave robot dynamics. We modify the slave and master robot dynamics by local adaptive controllers in such a way that both the master and the slave virtual robot dynamics are the same. This way, the hand force applied (thus felt) by the operator on the (virtual) master robot becomes equal to the force applied on the (virtual) slave robot. Therefore, the framework gives the operator a sense of being virtually present at the remote environment and using his/her tool to manipulate the environment.

The objective is to design the control inputs $\tau_{m}(t)$ and $\tau_{s}(t)$ in discrete-time such that $x_{s}(t) \rightarrow x_{m}(t)$ when the slave 
robot is in free-motion and $f_{e}(t) \rightarrow R f_{h}(t)$, for some scaling factor $R$, when the slave robot end-effector is in contact with a hard surface. These objectives must be satisfied when there is communication time-delay between the master robot and the slave robot. The time-delay can be specified as forward communication time-delay and backward communication time-delay. The forward communication time-delay can be represented in number of time-steps, namely, as $d_{1}$ where $\left(d_{1}-1\right) T \leq t_{1} \leq d_{1} T$ with $t_{1}$ being the actual time-delay and $T$ being the sampling-period. Similary, the backward communication time-delay can be represented as $d_{2}$ time-steps.

\section{FIXED CONTROLLER DESIGN}

In order to design the controller, the problem will be divided into two parts: 1 . Local adaptive controllers that cancel the nonlinear dynamics $Y(q, \dot{q}, \ddot{q}) \theta$ and impose the impedence $M \ddot{x}+B \dot{x}=f$, where $f$ is a fictitious control, at the end-effectors, 2. Design the fictitious control $f$ such that $\lim _{t \rightarrow \infty}\left\|x_{s}(t)-x_{m}(t)\right\| \rightarrow 0$ when the slave robot is in free-motion and $\lim _{t \rightarrow \infty} \| f_{s}(t)-$ $R f_{h}(t) \| \rightarrow 0$ when the slave robot end-effector is in contact with a surface. During contact with a surface, $\lim _{t \rightarrow \infty}\left\|f_{s}(t)-R f_{h}(t)\right\| \rightarrow 0$ would imply that $\lim _{t \rightarrow \infty}\left\|f_{e}(t)-R f_{m}(t)\right\| \rightarrow 0$, thus, the contact force $f_{e}(t)$ will be reflected back to the operator in the form of $f_{m}(t)$.

\subsection{Local Controller Design}

Local controller design involves no interaction between the master robot and slave robot, therefore, there will exist no time-delay in any of the signals. Consider the system (7) and (8). Since this will be a discrete-time implementation any time dependent function $\rho(t)$ will be replaced with $\rho_{k}$ where $k$ is the index of the sampling instant, also, for convenience let $Y_{m, k} \equiv Y_{m}\left(q_{m}, \dot{q}_{m}, \ddot{q}_{m}\right)$, $Y_{s, k} \equiv Y_{s}\left(q_{s}, \dot{q}_{s}, \ddot{q}_{s}\right), J_{m, k} \equiv J_{m}\left(q_{m}\right)$ and $J_{s, k} \equiv J_{s}\left(q_{s}\right)$. If the parameters of the robots and the contact forces are known then select the control law as

$$
\begin{gathered}
\tau_{m, k}=Y_{m, k} \theta_{m}-J_{m, k}^{T}\left(M \ddot{x}_{m, k}+B \dot{x}_{m, k}+f_{m, k}\right) \\
\tau_{s, k}=Y_{s, k} \theta_{s}-J_{s, k}^{T}\left(R M \ddot{x}_{s, k}+R B \dot{x}_{s, k}-f_{s, k}\right)
\end{gathered}
$$

where $R$ is a diagonal positive-definite constant matrix used for scaling the environmental contact forces. The mass matrix $M$ and damping matrix $B$ are selected to reflect a desired impedence of the slave robot. Substituting the control laws (9) and (10) into (7) and (8) to obtain the closed-loop system of the master robot as

$$
-J_{m, k}^{T}\left(M \ddot{x}_{m, k}+B \dot{x}_{m, k}+f_{m, k}-f_{h, k}\right)=0
$$

and slave robot as

$$
-J_{s, k}^{T}\left(R M \ddot{x}_{s, k}+R B \dot{x}_{s, k}-f_{s, k}+f_{e, k}\right)=0,
$$

ensuring that the desired impedences are imposed at the end-effector of the master and slave robots.

Remark 1. Unlike position and velocity, the acceleration terms in (9) and (10) may not be easily available. However, advances in sensor technology such as that which is shown in [28], [29] and [30] have made it possible for the accurate measurement of accelerations and forces and there have been controllers proposed in the literature for stable teleoperation that assumes such measurements are available [31], [32], [9]. However, depending on the application, the need for filtering may introduce robustness issues. In this paper it is assumed that this is not the case. A modified version of the proposed controller that does not require these measurements is the topic of future research.

\subsection{Fictitious Controller Design}

Fictitious controller design involves interaction between the master robot and slave robot and, therefore, will be handeled keeping in mind the forward and backward communication time-delay. Now, consider the dynamics at the end-effectors given by

$$
\begin{gathered}
M \ddot{x}_{m, k}+B \dot{x}_{m, k}=-f_{m, k}+f_{h, k} \\
R M \ddot{x}_{s, k}+R B \dot{x}_{s, k}=f_{s, k}-f_{e, k}
\end{gathered}
$$

To proceed with the selection of the fictitious control inputs $f_{m, k}$ and $f_{s, k}$ the system (13) and (14) is written in the sampled-data form

$$
\begin{array}{r}
\chi_{m, k+1}=\Phi \chi_{m, k}-\Gamma f_{m, k}+\Gamma f_{h, k}, \\
\chi_{s, k+1}=\Phi \chi_{s, k}+\Gamma R^{-1} f_{s, k}-\Gamma R^{-1} f_{e, k}
\end{array}
$$

where $\Phi, \Gamma$ are the sampled-data state and input matrices computed from

$$
\begin{aligned}
& \Phi=\exp \left(\left[\begin{array}{cc}
\mathbf{0} & \mathbf{1} \\
\mathbf{0} & -M^{-1} B
\end{array}\right] T\right) \\
& \Gamma=\int_{0}^{T} \exp \left(\left[\begin{array}{cc}
\mathbf{0} & \mathbf{1} \\
\mathbf{0} & -M^{-1} B
\end{array}\right] \sigma\right)\left[\begin{array}{c}
\mathbf{0} \\
M^{-1}
\end{array}\right] d \sigma
\end{aligned}
$$

where $T$ is the sampling-time. In (16), the fictitious control $f_{s, k}$ is selected as a PD-controller and since there may exist communication time-delays between the master and slave robots the PD-controller is given as

$$
\begin{aligned}
f_{s, k} & =K_{s}\left(x_{m, k-d_{1}}-x_{s, k}\right)+B_{s}\left(\dot{x}_{m, k-d_{1}}-\dot{x}_{s, k}\right) \\
& =\Theta_{s}\left(\chi_{m, k-d_{1}}-\chi_{s, k}\right)
\end{aligned}
$$

where $K_{s}$ and $B_{s}$ are the PD-controller gains which act as stiffness and damping, and $\Theta_{s} \equiv \operatorname{diag}\left(K_{s}, B_{s}\right)$. Since the parameters of the system (15) and (16) are known, the gains of the controller (17) can be selected so that $\lim _{t \rightarrow \infty}\left\|\chi_{m, k-d_{1}}-\chi_{s, k}\right\|=0$ when the slave robot is in free-motion, according to certain control specifications imposed by the task.

Remark 2. Note that in the case when $\alpha_{0} \neq 0$ in (5), there will be a constant steady steady error in the tracking of $x_{m, k}$ by $x_{s, k}$. This steady state error can be eliminated by including integral action in $f_{s, k}$.

The dynamics of the fictitious slave input force $f_{s, k}$ can be found by substituting (15) and (16) into a single time-step 
shifted (17) as

$$
\begin{aligned}
f_{s, k+1} & =\Theta_{s}\left(\chi_{m, k-d_{1}+1}-\chi_{s, k+1}\right) \\
& =\Theta_{s} \Phi \chi_{m, k-d_{1}}-\Theta_{s} \Phi \chi_{s, k}-\Theta_{s} \Gamma R^{-1} f_{s, k} \\
& +\Theta_{s} \Gamma f_{h, k-d_{1}}+\Theta_{s} \Gamma R^{-1} f_{e, k}-\Theta_{s} \Gamma f_{m, k-d_{1}} .
\end{aligned}
$$

Substitution of (6) and (17) in (18) results in the final form of the fictitious slave input force dynamics

$$
\begin{aligned}
& f_{s, k+1}=\Theta_{s}\left(\Phi-\Gamma R^{-1} \Theta_{s}\right)\left(\chi_{m, k-d_{1}}-\chi_{s, k}\right) \\
& +\Theta_{s} \Gamma f_{h, k-d_{1}}+\Theta_{s} \Gamma R^{-1} \Theta_{e} \chi_{s, k}-\Theta_{s} \Gamma f_{m, k-d_{1}} .
\end{aligned}
$$

Let $\Theta_{\phi} \equiv \Theta_{s}\left(\Phi-\Gamma R^{-1} \Theta_{s}\right)$ and $\Theta_{\gamma} \equiv \Theta_{s} \Gamma$ then (19) can be rewritten as

$$
\begin{aligned}
f_{s, k+1} & =\Theta_{\phi}\left(\chi_{m, k-d_{1}}-\chi_{s, k}\right)+\Theta_{\gamma} f_{h, k-d_{1}} \\
& +\Theta_{\gamma} R^{-1} f_{e, k}-\Theta_{\gamma} f_{m, k-d_{1}} .
\end{aligned}
$$

Remark 3. During contact with a hard surface, the velocity and acceleration of the slave robot would be approximately zero and, therefore, $f_{s, k} \approx f_{e, k}$. Thus, $\lim _{k \rightarrow \infty}\left\|f_{s, k}-R f_{h, k}\right\| \rightarrow 0$ would imply that $\lim _{k \rightarrow \infty}\left\|f_{e, k}-R f_{h, k}\right\| \rightarrow 0$.

In order to achieve $\left\|f_{s, k+1}-R f_{h, k-d_{1}}\right\| \rightarrow 0$, note that in (20) the control input, $f_{m, k}$, is delayed by $d_{1}$ time-steps and, therefore, the control law design will require future states as shown below

$$
\begin{aligned}
& f_{m, k}=\left(I+\Theta_{\gamma}^{-1} R\right) f_{h, k}+\Theta_{\gamma}^{-1} \Theta_{\phi} \chi_{m, k} \\
& -\Theta_{\gamma}^{-1} \Theta_{\phi} \chi_{s, k+d_{1}}+R^{-1} f_{e, k+d_{1}}
\end{aligned}
$$

which is obtained by substituting (20) in $f_{s, k+1}-$ $R f_{h, k-d_{1}}=0$ and solving for $f_{m, k}$. Since the future value of $\chi_{s, k}$ and $f_{e, k}$ are not available these will be estimated from (16). Substitution of (17) in (16) it is obtained that

$$
\begin{aligned}
& \chi_{s, k+1}=\left(\Phi-\Gamma\left(\Theta_{s}+R^{-1} \Theta_{e}\right)\right) \chi_{s, k} \\
& +\Gamma \Theta_{s} \chi_{m, k-d_{1}} .
\end{aligned}
$$

Writing (22) repeatedly it is obtained that

$$
\chi_{s, k+d}=\Phi_{e}^{d} \chi_{s, k}+\sum_{i=0}^{d-1} \Phi_{e}^{d-1-i} \Gamma \Theta_{s} \chi_{m, k-d_{1}+i}
$$

where $\Phi_{e} \equiv\left(\Phi-\Gamma\left(\Theta_{s}+R^{-1} \Theta_{e}\right)\right.$ and $d=d_{1}+d_{2}$. Here, $d_{2}$ is the backward communication delay in timesteps. Note that since there exists a backward communication delay between the slave and master robots, the future estimate of $\chi_{s, k}$ can be computed only using the availabe measurement $\chi_{s, k-d_{2}}$. Therefore, the future estimate $\chi_{s, k+d_{1}}$ is given as

$$
\chi_{s, k+d_{1}}=\Phi_{s}^{d} \chi_{s, k-d_{2}}+\sum_{i=0}^{d-1} \Phi_{s}^{d-1-i} \Gamma \Theta_{s} \chi_{m, k-d+i}
$$

All the terms on the right-hand-side of (24) are available and, therefore, the control law (21) becomes

$$
\begin{aligned}
& f_{m, k}=\left(I+\Theta_{\gamma}^{-1} R\right) f_{h, k}+\Theta_{\gamma}^{-1} \Theta_{\phi} \chi_{m, k} \\
& -\Theta_{\gamma}^{-1} \Theta_{\phi}\left[\Phi_{e}^{d} \chi_{s, k-d_{2}}+\sum_{i=0}^{d-1} \Phi_{e}^{d-1-i} \Gamma \Theta_{s} \chi_{m, k-d+i}\right] .
\end{aligned}
$$

Controller (25) is in causal form and should reflect the force on the slave robot accurately.

\section{ADAPTIVE CONTROLLER DESIGN}

In this section, the adaptive controller design is introduced as well as the necessary modifications to the fictitious controller (25) to ensure asymptotic stability.

\subsection{Local Adaptive Controller}

When the robot parameters $\theta_{m}$ and $\theta_{s}$ are uncertain, then the control laws (9) and (10) can be modified to the form

$$
\begin{aligned}
& \tau_{m, k}=Y_{m, k} \hat{\theta}_{m, k}-J_{m, k}^{T}\left(M \ddot{x}_{m, k}+B \dot{x}_{m, k}+f_{m, k}\right. \\
& \left.-f_{h, k}\right) \\
& \tau_{s, k}=Y_{s, k} \hat{\theta}_{s, k}-J_{s, k}^{T}\left(R M \ddot{x}_{s, k}+R B \dot{x}_{s, k}-f_{s, k}\right. \\
& \left.\quad+f_{e, k}\right)
\end{aligned}
$$

where $\hat{\theta}_{m, k}$ and $\hat{\theta}_{s, k}$ are the estimates of $\theta_{m}$ and $\theta_{s}$ repectively.

Substituting the control laws (26) and (27) into (7) and (8) to obtain the closed-loop system of the master robot as

$$
\begin{aligned}
& Y_{m, k} \tilde{\theta}_{m, k}=-J_{m, k}^{T}\left(M \ddot{x}_{m, k}+B \dot{x}_{m, k}+f_{m, k}\right. \\
& \left.-f_{h, k}\right)
\end{aligned}
$$

and slave robot as

$$
\begin{aligned}
& Y_{s, k} \tilde{\theta}_{s, k-d_{1}}=-J_{s, k}^{T}\left(R M \ddot{x}_{s, k}+R B \dot{x}_{s, k}-f_{s, k}\right. \\
& \left.+f_{e, k}\right)
\end{aligned}
$$

where $\tilde{\theta}_{m, k}=\theta_{m}-\hat{\theta}_{m, k}$ and $\tilde{\theta}_{s, k}=\theta_{s}-\hat{\theta}_{s, k}$ are the parameter estimation errors. From (28) and (29) the adaptation laws are formulated as

$$
\begin{gathered}
\hat{\theta}_{m, k+1}=\hat{\theta}_{m, k}-P_{m, k+1} Y_{m, k}^{T} J_{m, k}^{T} z_{m, k} \\
\hat{\theta}_{s, k+1}=\hat{\theta}_{s, k}-P_{s, k+1} Y_{s, k}^{T} J_{s, k}^{T} z_{s, k}
\end{gathered}
$$

where $z_{m, k} \equiv M \ddot{x}_{m, k}+B \dot{x}_{m, k}+f_{m, k}-f_{h, k}, z_{s, k} \equiv$ $R M \ddot{x}_{s, k}+R B \dot{x}_{s, k}-f_{s, k}+f_{e, k}$ and $d_{1}$ is the forward timedelay in time-steps. The matrices $P_{m, k}, P_{s, k}$ are symmetric positive definite matrices computed as

$$
\begin{gathered}
P_{m, k+1}=P_{m, k}-P_{m, k} Y_{m, k}^{T}(I \\
\left.+Y_{m, k} P_{m, k} Y_{m, k}^{T}\right)^{-1} Y_{m, k} P_{m, k} \\
P_{s, k+1}=P_{s, k-d_{1}}-P_{s, k-d_{1}} Y_{s, k}^{T}(I \\
\left.+Y_{s, k} P_{s, k-d_{1}} Y_{s, k}^{T}\right)^{-1} Y_{s, k} P_{s, k-d_{1}} .
\end{gathered}
$$

The covariance matrix $P$ has some useful properties, [27] Property 2. $P_{k+1}^{-1}=P_{k}^{-1}+Y_{k}^{T} Y_{k}$

Property 3. $Y_{k} P_{k+1} Y_{k}^{T}=\left(I+Y_{k} P_{k} Y_{k}^{T}\right)^{-1} Y_{k} P_{k} Y_{k}^{T}$ The adaptation laws (30) and (31) are implemented to guarantee that $\lim _{t \rightarrow \infty}\left\|z_{m, k}\right\| \rightarrow 0$ and $\lim _{t \rightarrow \infty}\left\|z_{s, k}\right\| \rightarrow 0$ or, in other words, the desired impedence is imposed at the end-effectors of both the master and slave robots. The asymptotic stability of the closed-loop system (28) and (29) 
with the adaptation laws (30) and (31) will not be presented here due to space constraints. However, these proofs will be included in the journal version of the paper.

Next, consider the external force model (6), if $\Theta_{e}$ is uncertain then

$$
\hat{f}_{e, k}=\hat{K}_{e, k} x_{s, k}+\hat{B}_{e, k} \dot{x}_{s, k}=\hat{\Theta}_{e, k} \chi_{s, k}
$$

where $\hat{\Theta}_{e, k}$ is the estimate of $\Theta_{e}$. Since $f_{e, k}$ is measured, it is possible to write

$$
f_{e, k}-\hat{f}_{e, k}=\Theta_{e} \chi_{s, k}-\hat{\Theta}_{e, k} \chi_{s, k}=\tilde{\Theta}_{e, k} \chi_{s, k} .
$$

An adaptation law can be formulated for $\hat{\Theta}_{e, k}$ as follows

$$
\begin{gathered}
\hat{\Theta}_{e, k+d_{2}}=\hat{\Theta}_{e, k}-P_{e, k+d_{2}} \chi_{s, k}^{T}\left(f_{e, k}-\hat{f}_{e, k}\right) \\
P_{e, k+d_{2}}=P_{e, k}-\frac{P_{e, k} \chi_{s, k} \chi_{s, k}^{T} P_{e, k}}{1+\chi_{s, k}^{T} P_{e, k} \chi_{s, k}} .
\end{gathered}
$$

\subsection{Modified Fictitious Controller}

Consider the dynamics at the end-effectors given by

$$
\begin{gathered}
M \ddot{x}_{m, k}+B \dot{x}_{m, k}=-f_{m, k}+f_{h, k}+\delta_{m, k} \\
R M \ddot{x}_{s, k}+R B \dot{x}_{s, k}=f_{s, k}-\hat{f}_{e, k}+\delta_{s, k}+\delta_{e, k}
\end{gathered}
$$

where $\delta_{m, k}, \delta_{s, k}$ and $\delta_{e, k}$ are the errors incurred from the adaptive laws (30), (31) and (36). In sampled-data form the system (38) and (39) are written as

$$
\begin{aligned}
& \chi_{m, k+1}=\Phi \chi_{m, k}-\Gamma f_{m, k}+\Gamma f_{h, k}+\Gamma \delta_{m, k}, \\
& \chi_{s, k+1}=\Phi \chi_{s, k}+\Gamma R^{-1} f_{s, k}-\Gamma R^{-1} \hat{\Theta}_{e, k} \chi_{s, k} \\
& +\Gamma R^{-1}\left(\delta_{s, k}+\delta_{e, k}\right)
\end{aligned}
$$

The slave input force dynamics (19) is modified to

$$
\begin{aligned}
& f_{s, k+1}=\Theta_{\phi}\left(\chi_{m, k-d_{1}}-\chi_{s, k}\right)+\Theta_{s} \Gamma f_{h, k-d_{1}} \\
& +\Theta_{s} \Gamma R^{-1} \hat{f}_{e, k}-\Theta_{s} \Gamma R^{-1} \delta_{s, k}+\Theta_{s} \Gamma \delta_{m, k-d_{1}} \\
& -\Theta_{s} \Gamma f_{m, k-d_{1}} .
\end{aligned}
$$

In order to achieve $\lim _{t \rightarrow \infty}\left\|f_{s, k+1}-R f_{h, k-d_{1}}\right\| \rightarrow 0$, the control law (21) remains the same. Consider $\chi_{s, k}$, the future estimate is computed the same way as in (23). Substituting (17) and (34) in (16) it is obtained that

$$
\begin{aligned}
& \chi_{s, k+d}=\Phi_{s}^{d} \chi_{s, k} \\
& +\sum_{i=0}^{d-1} \Phi_{s}^{d-1-i}\left(\Gamma \Theta_{s} \chi_{m, k-d_{1}+i}-\Gamma R^{-1} \hat{f}_{e, k+i}\right. \\
& \left.+\Gamma R^{-1} \delta_{e, k+i}\right)
\end{aligned}
$$

where $\Phi_{s} \equiv\left(\Phi-\Gamma \Theta_{s}\right)$ and $\delta_{e, k}$ is the error $\tilde{f}_{e, k}$. Writing (43) repeatedly it is obtained that

$$
\begin{aligned}
& \chi_{s, k+d}=\left(\prod_{j=0}^{d-1} \Phi_{e, k-d_{1}+j}\right) \chi_{s, k} \\
& +\sum_{i=0}^{d-1}\left(\prod_{j=i+1}^{d-1} \Phi_{e, k-d_{1}+j}\right) \Theta_{\gamma} \chi_{m, k-d_{1}+i} \\
& +\sum_{i=0}^{d-1}\left(\prod_{j=i+1}^{d-1} \hat{\Phi}_{e, k-d_{1}+j}\right) \Gamma\left(\delta_{e, k+i}+\delta_{s, k+i}\right)
\end{aligned}
$$

where $\hat{\Phi}_{e, k} \equiv\left[\Phi-\Gamma\left(\Theta_{s}+R \hat{\Theta}_{e, k}\right)\right]$ and $d=d_{1}+d_{2}$. Similar to (24), the future estimate of $\chi_{s, k}$ can be computed only using the availabe measurement $\chi_{s, k-d_{2}}$. Note that from (23) the future of the transient errors $\delta_{s, k}$ is needed to compute the future value of $\chi_{s, k+d_{1}}$. To circumvent, a future estimate of $\chi_{s, k+d_{1}}$ is given as

$$
\begin{aligned}
& \hat{\chi}_{s, k+d_{1}}=\left(\prod_{j=0}^{d-1} \Phi_{e, k-d+j}\right) \chi_{s, k-d_{2}} \\
& +\sum_{i=0}^{d-1}\left(\prod_{j=i+1}^{d-1} \Phi_{e, k-d+j}\right) \Theta_{\gamma} \chi_{m, k-d+i} .
\end{aligned}
$$

All the terms on the right-hand-side of (45) are available and, therefore, the control law (25) becomes

$$
\begin{aligned}
& f_{m, k}=\left(I+\Theta_{\gamma}^{-1}\right) f_{h, k}+\Theta_{\gamma}^{-1} \Theta_{\phi} \chi_{m, k} \\
& -\left(\Theta_{\gamma}^{-1} \Theta_{\phi}-R \hat{\Theta}_{e, k}\right) \hat{\chi}_{s, k+d_{1}} .
\end{aligned}
$$

Thus, the controller (46) is computed in causal form.

Remark 4. Dropping the transient error term $\delta_{s, k}$ does not undermine the stability of the system and adds a transient error term to the force tracking error $\left\|f_{s, k}-\hat{f}_{h, k-d_{1}}\right\|$ that converges to zero aymptotically. The proof is not provided here due to space limitations but will be included in the journal version of the paper.

\section{SUMMARY}

In this paper, a new adaptive approach was proposed for the stable operation of a telerobotic systems with force feedback, in the presence of communication time delays and parameteric uncertainties. The proof of stability is not presented here due to space constraints. The proof will be included in the journal version of the paper.

\section{REFERENCES}

[1] G. Niemeyer, "Using wave variables in time-delayed force reflecting teleoperation," Ph.D. dissertation, Massachusetts Institute of Technology, 1996.

[2] G. Niemeyer and J.-J. Slotine, "Stable adaptive teleoperation," IEEE Journal of Oceanic Engineering, vol. 16, no. 1, pp. 152-162, 1991.

[3] _ - "Using wave variables for system analysis and robot control," Robotics and Automation, vol. 2, pp. 1619-1625, 1991

[4] H. Arioui, A. Kheddar, and S. Mammar, "A predictive wave-based approach for time delayed virtual environments environments haptics systems," in Proceedings of the IEEE InternationalWorkshop on Robot and Human Interactive Communication, Berlin, Germany, 2002.

[5] S. Ganjefar, H. Momeni, and F. Janabi-Sharifi, "Teleoperation systems design using augmented wave-variables and smith predictor method for reducing time-delay effects," in Proceedings of the International Symposium on Intelligent Control, Vancouver, Canada, 2002.

[6] D. Lee and M. Spong, "Passive bilateral teleoperation with constant time delay," IEEE Transactions on Robotics, vol. 22, no. 2, pp. 269-281, 2006. 